\title{
STOCHASTIC POST-PROCESSING OF THE DETERMINISTIC BOUNDARY ELEMENT MODELLING OF THE TRANSIENT ELECTRIC FIELD FROM GPR DIPOLE ANTENNA PROPAGATING THROUGH LOWER HALF-SPACE
}

\author{
D. POLJAK ${ }^{1}, \mathrm{~S} . \mathrm{SESNIC}^{1}$ S. LALLECHERE ${ }^{2} \&$ K. EL KHAMLICHI DRISSI ${ }^{2}$ \\ ${ }^{1}$ University of Split, Split, Croatia. \\ ${ }^{2}$ Blaise Pascal University, Clermont-Ferrand, France.
}

\begin{abstract}
The paper deals with time domain-deterministic stochastic assessment of a transient electric field generated by a ground penetrating radar (GPR) dipole antenna and transmitted into a lower half-space. The deterministic time domain formulation is based on the space-time Hallen integral equation for half-space problems. The Hallen equation is solved via the Galerkin-Bubnov variant of the Indirect Boundary Element Method (GB-IBEM) and the space-time current distribution along the dipole antenna is obtained, thus providing the field calculation. The field transmitted into the lower medium is obtained by solving the corresponding field integrals.

As GPR systems are subjected to a rather complex environment, some input parameters, for example the antenna height over ground or earth properties, are partly or entirely unknown and, therefore, a simple stochastic collocation (SC) method is used to properly access relevant statistics about GPR time responses. The SC approach also aids in the assessment of corresponding confidence intervals from the set of obtained numerical results. The expansion of statistical output in terms of mean and variance over a polynomial basis, via the SC method, is shown to be a robust and efficient approach providing a satisfactory convergence rate.

Keywords: deterministic boundary element modelling, ground penetrating radar, hallen integral equation, stochastic collocation method, time domain, transmitted field.
\end{abstract}

\section{INTRODUCTION}

There has been a continuous interest in the analysis of GPR (Ground Penetrating Radar) systems and related applications, or example in civil engineering [1]. The element of particular importance for a GPR system operation is an antenna [2].

Many efficient GPR antenna models are based on the Finite Difference Time Domain (FDTD) simulations, e.g. [3-5].

Contrary to the widely used FDTD approach, this paper presents an approach for the evaluation of transient electric field transmitted into the lower half-space due to the GPR dipole antenna via the Boundary Element Method (BEM) combined with the stochastic collocation (SC) approach.

The deterministic model is based on the direct time-domain analysis of a single straight wire above a lossy half-space and the corresponding space-time Hallen integral equation [6-8]. The presence of the air-earth interface is taken into account via the space-time Fresnel reflection coefficient. The space-time Hallen equation is solved via the Galerkin-Bubnov variant of the Indirect Boundary Element Method (GB-IBEM) [6-8]. A comparison of the FDTD approach and the time domain integral equation method using the approach promoted in [9] has been carried out recently [10]. 
Furthermore, a novel formula for the transient field transmitted into a dielectric half-space is reported in [9]. The influence of a two-media interface is taken into account via the simplified space-time transmission coefficient arising from the Modified Image Theory (MIT). The corresponding transmitted field is obtained from the related field integrals using the boundary element formalism, as well.

On the other hand, the uncertainty of the input parameters inevitably leads to the uncertainty in the assessment of the transient field transmitted through the lower medium.

A novel approach to properly quantify and handle the uncertainties, presented in this work, is related to the use of the SC technique [11, 12], which combines deterministic electromagnetic techniques with certain statistical methods.

Some illustrative numerical results for the transient transmitted field pertaining to certain statistical moments are given in the paper, as well.

\section{FORMULATION: DETERMINISTIC AND STOCHASTIC APPROACH}

This section outlines the formulation, deterministic boundary element procedures and the SC approach.

\subsection{Deterministic modelling}

The horizontal GPR dipole antenna of length $L$, radius $a$, located at height $h$ and radiating above a lossy half-space is depicted in Fig. 1. The antenna is excited by an equivalent voltage source $V_{\mathrm{g}}$.

The transient current on the horizontal straight thin wire over a lossy half-space illuminated by a tangential incident electric field $E_{x}^{i n c}$, existing in the antenna feed-gap is governed by the space-time Hallen integral equation [6]:

$$
\begin{aligned}
& \int_{0}^{L} \frac{I\left(x^{\prime}, t-R_{a} / c\right)}{4 \pi R_{a}} d x^{\prime}-\int_{-\infty}^{t} \int_{0}^{L} r(\theta, \tau) \frac{I\left(x^{\prime}, t-R_{d}^{*} / c-\tau\right)}{4 \pi R_{d}^{*}} d x^{\prime} d \tau= \\
& =F_{0}\left(t-\frac{x}{c}\right)+F_{L}\left(t-\frac{L-x}{c}\right)+\frac{1}{2 Z_{0}} \int_{0}^{L} E_{x}^{i n c}\left(x^{\prime}, t-\frac{\left|x-x^{\prime}\right|}{c}\right) d x^{\prime}
\end{aligned}
$$

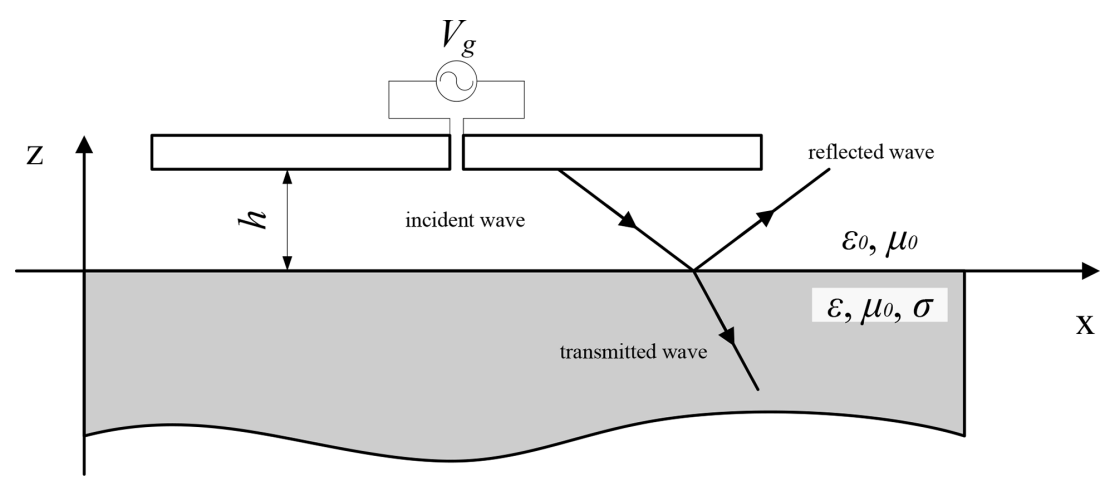

Figure 1: Centre-fed GPR dipole antenna. 
Where $I(x, t)$ is the unknown axial current along the wire, $c$ is the velocity of light, $Z_{0}$ is the free-space wave impedance, while $R_{a}$ and $R_{a}{ }^{*}$ denote the distances between observation point $x$ and source point $x$ ' on actual and image wire, respectively. Unknown functions $F_{0}$ and $F_{L}$ account for the multiple reflections from the wire ends, and can be determined by assuming the zero current at the wire ends [6-8]. The influence of the ground is taken into account via the reflection coefficient (RC) function $r(\theta, \mathrm{t})[6-8]$ :

$$
r(\theta, t)=K \delta(t)+\frac{4 \beta}{1-\beta^{2}} \frac{e^{-\alpha t}}{t} \sum_{n=1}^{\infty}(-1)^{n+1} n K^{n} I_{n}(\alpha t)
$$

where

$$
K=\frac{1-\beta}{1+\beta} ; \beta=\frac{\sqrt{\varepsilon_{r}-\sin ^{2} \theta}}{\varepsilon_{r} \cos \theta} ; \alpha=\frac{\sigma}{2 \varepsilon} ; \theta=\operatorname{arctg} \frac{|x-x|}{2 h},
$$

Note that $\delta(t)$ is the Dirac impulse, and $I_{n}(t)$ is the $n$th order modified Bessel function of the first kind.

In the case of a transmitting dipole-antenna the voltage generator differs from zero only in the feed-gap area: $x_{g}-\Delta l_{g}<x<x_{g}+\Delta l_{g}$ the incident field can be written in terms of the Dirac impulse notation:

$$
E_{x}^{i n c}(x, t)=V_{g}(t) \delta\left(x-x_{g}\right)
$$

where $V_{g}$ denotes the voltage generator and $x_{g}=L / 2$.

For sufficiently small gap dimensions (delta-function generator) one has:

$$
\int_{x_{g}-\Delta l_{g}}^{x_{g}+\Delta l_{g}} E_{x}^{i n c}\left(x^{\prime}, t-\frac{\left|x-x^{\prime}\right|}{c}\right) d x^{\prime}=V_{g}\left(x^{\prime}, t-\frac{\left|x-x_{g}\right|}{c}\right)
$$

The Hallen eqn (1) is solved via the space-time version of GB-IBEM [8] forcing the zero current at the free ends of the wire and with the initial conditions requiring the wire not to be excited before the certain instant $t=t_{0}$. Once the space-time dependent current distribution along the dipole antenna is determined, the related electric field penetrating through the two-media interface can be calculated.

A space-time expression for the transient field transmitted in a lower lossy half-space has been reporetd in [9] and is given by:

$$
E_{x}^{t r}(r, t)=\frac{\mu_{0}}{4 \pi} \int_{-\infty}^{t} \int_{0}^{L} \Gamma_{t r}^{M I T}(\theta, \tau) \frac{\partial I\left(x^{\prime}, t-R^{\prime \prime} / v-\tau\right)}{\partial t} \frac{e^{-\frac{1}{\tau_{g}} \frac{R^{\prime \prime}}{v}}}{R^{\prime \prime}} d x^{\prime} d \tau
$$

where $v$ stands for the velocity of wave propagation in the lower medium while $R$ " is the distance from the dipole antenna to the observation point located in the lower half-space.

The transmitting effects of the air-ground interface are taken into account via a simplified space-time transmission coefficient arising from the MIT [9]

$$
\Gamma_{t r}^{M I T}=\frac{\tau_{g}}{\tau_{g 2}}\left[\delta(t)+\frac{1}{\tau_{g 2}}\left(1-\frac{\tau_{g}}{\tau_{g 2}}\right) e^{-t / \tau_{g}}\right]
$$


where:

$$
\tau_{g}=\frac{2 \varepsilon_{r}}{\sigma} \varepsilon_{0}, \quad \tau_{g 2}=\frac{\varepsilon_{r}+1}{\sigma} \varepsilon_{0}, \quad t "=t-\frac{R_{d}^{\prime}}{v}-\tau
$$

Note that in the case of dielectric half-space (6) simplifies into:

$$
E_{x}^{t r}(r, t)=\frac{\mu_{0}}{4 \pi} \int_{0}^{L} \Gamma_{t r}^{M I T}(\theta) \frac{\partial I\left(x^{\prime}, t-R_{d}^{\prime \prime} / v\right)}{\partial t} \frac{1}{R_{d}^{\prime \prime}} d x^{\prime}
$$

The field transmitted in a dielectric and/or lossy half-space, respectively, can be calculated by using the boundary element formalism described in [9].

Using the local approximation for current [8]:

$$
I\left(x^{\prime}, t^{\prime}\right)=\{f\}^{T}\left\{I\left(t^{\prime}\right)\right\}
$$

where $\left\{I\left(t^{\prime}\right)\right\}$ is the vector containing unknown coefficients, and linear shape functtions are given by [3]

$$
f_{r}\left(x^{\prime}\right)=\frac{x_{r+1}-x^{\prime}}{x_{r+1}-x_{r}} f_{r+1}\left(x^{\prime}\right)=\frac{x^{\prime}-x_{r}}{x_{r+1}-x_{r}}
$$

After performing the space discretization one has

$$
E_{x, i}^{t r}(r, t)=\frac{\mu_{0}}{4 \pi} \Gamma_{t r}^{M I T}(\theta)\left[\int_{\Delta I_{i}} \frac{\partial}{\partial t}\{I\}_{\mid t^{\prime \prime}=t-R^{\prime \prime} / v}\left\{f^{\prime \prime}\right\}^{T} \frac{1}{R_{d}^{\prime \prime}} d x^{\prime}\right]
$$

while the temporal discretization finally yields

$$
E_{x, i}^{t r}(r, t)=\frac{\mu_{0}}{4 \pi} \Gamma_{t r}^{M I T}(\theta)\left[\int_{\Delta \Delta_{i}} \frac{I_{i 1}^{m+1}-I_{i 1}^{m}}{\Delta t} f_{i 1}\left(x^{\prime}\right) \frac{1}{R_{d}^{\prime \prime}} d x^{\prime}+\int_{\Delta \Delta_{i}} \frac{I_{i 2}^{m+1}-I_{i 2}^{m}}{\Delta t} f_{i 2}\left(x^{\prime}\right) \frac{1}{R_{d}^{\prime \prime}} d x^{\prime}\right]
$$

An extension of the BEM procedure to lossy media will be reported in future work.

\subsection{Stochastic analysis}

Once the time domain deterministic modelling via GB-IBEM is carried out, a stochastic processing of the obtained numerical results is undertaken via the SC technique [11]. The fundamental principle of the SC technique is to use the polynomial approximation of the certain output $E$ of interest for $N$ given random parameters. The random parameter $Z$ is defined as [11, 12]:

$$
Z=Z^{0}+\widehat{u}
$$

where $Z^{0}$ is the initial (mean) value of a considered parameter, while $\hat{u}$ is the random variable $(\mathrm{RV})$ with assigned statistical distribution.

The function $t \rightarrow E\left(Z^{0}, t\right)$ is expanded over a stochastic space using the Lagrangian basis functions set $[11,12]$ :

$$
E\left(Z^{0}, t\right)=\sum_{i=1}^{n} E_{i}\left(Z^{0}\right) L_{i}(t)
$$

where $E$ is the output of interest (electric field in this work) and $L_{\mathrm{i}}(\mathrm{t})$ are Lagrange polynomials. 
The mean, variance and higher statistical moments can be obtained by undertaking stochastic analysis presented in $[11,12]$ for the case of single (one-dimensional) and multiple (multi-dimensional) random variable (RV), respectively [11].

More details related to this approach are available in $[11,12]$.

\section{COMPUTATIONAL EXAMPLES}

Computational examples are related to the horizontal dipole antenna of length $L=1 \mathrm{~m}$ and radius $a=6.74 \mathrm{~mm}$ radiating above a dielectric half-space. The dipole is driven at its centre by the Gaussian pulse voltage source:

$$
V(t)=V_{0} e^{-g^{2}\left(t-t_{0}\right)^{2}}
$$

with parameters: $V_{0}=1 \mathrm{~V}, g=1.5^{*} 10^{9} \mathrm{~s}^{-1}, t_{0}=1.43 \mathrm{~ns}$.

Figure 2 shows the transient electric field transmitted into the lower half-space in the broadside direction arising from the deterministic model computed at various depths. The permittivity of the lower medium is $\varepsilon_{r}=10$, while the antenna height is $h=0.1 \mathrm{~m}$. Figure 3 shows the influence of the various permittivity to the transient field at depth $d=0.5$ for the antenna height $h=0.1 \mathrm{~m}$. The influence of the parameter variation to the transient field in the sense of time delay, attenuation and changes in waveforms is visible in both cases.

Figure 4 shows the mean of the electric field inside the soil, for the following random variable: $\mathrm{RV} 1=h$ (antenna height above a soil), $\mathrm{RV} 2=\varepsilon_{\mathrm{r}}$ (soil permitivitty), RV3 $=d$ (field observation point). First, one random variable at a time is considered, as follows:

- RV1 - central location, $h^{0}=0.3 \mathrm{~m}$; uniformly distributed: $U[0.05 ; 0.55]$ (in metre)

- RV2 - initial value, $\varepsilon_{\mathrm{r}}{ }^{0}=15$; uniformly distributed: $U[2 ; 28]$

- RV3 - initial value, $d^{0}=1 \mathrm{~m}$; uniformly distributed: $U[0.5 ; 1.5]$ (in metre)

For SC convergence, $5 \mathrm{SC}$ points are required from 12 ns. Significant differences are noticeable between different scenarios with one random variable at a time, that is between RV1, RV2, RV3 models. Definitely, there is a need for $3 \mathrm{RVs}$ modelling at a time.

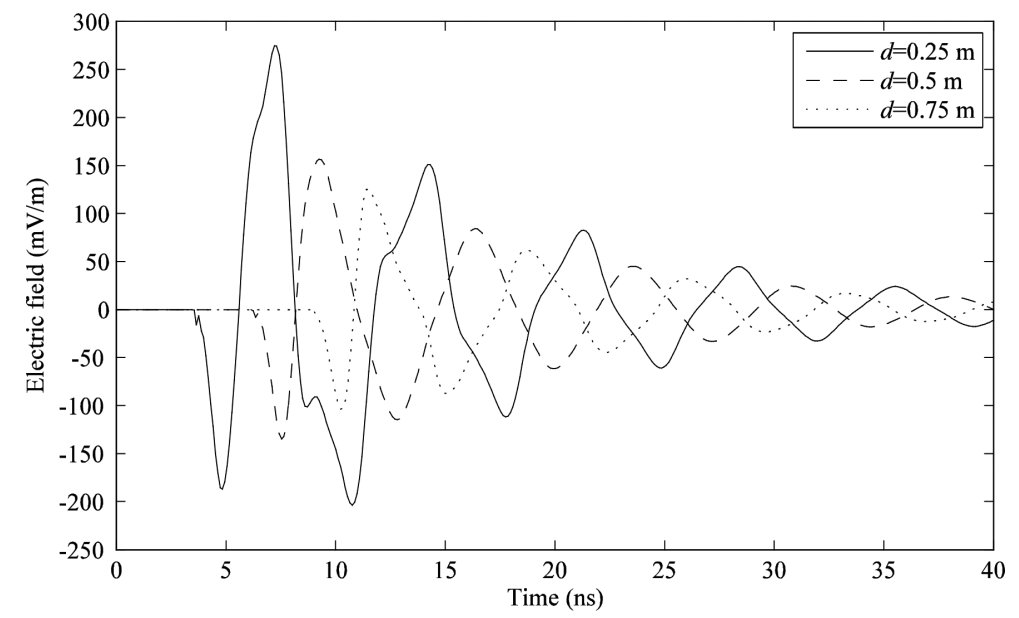

Figure 2: Transmitted electric field for various depths $\left(\varepsilon_{r}=10, h=0.1 \mathrm{~m}\right)$. 


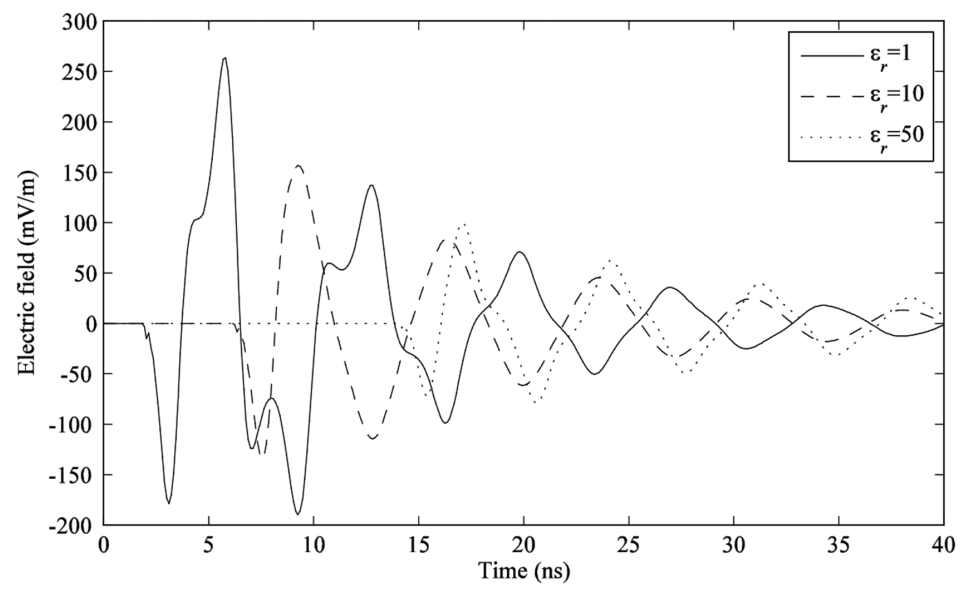

Figure 3: Transmitted electric field for various permittivity $(d=0.5, h=0.1 \mathrm{~m})$.

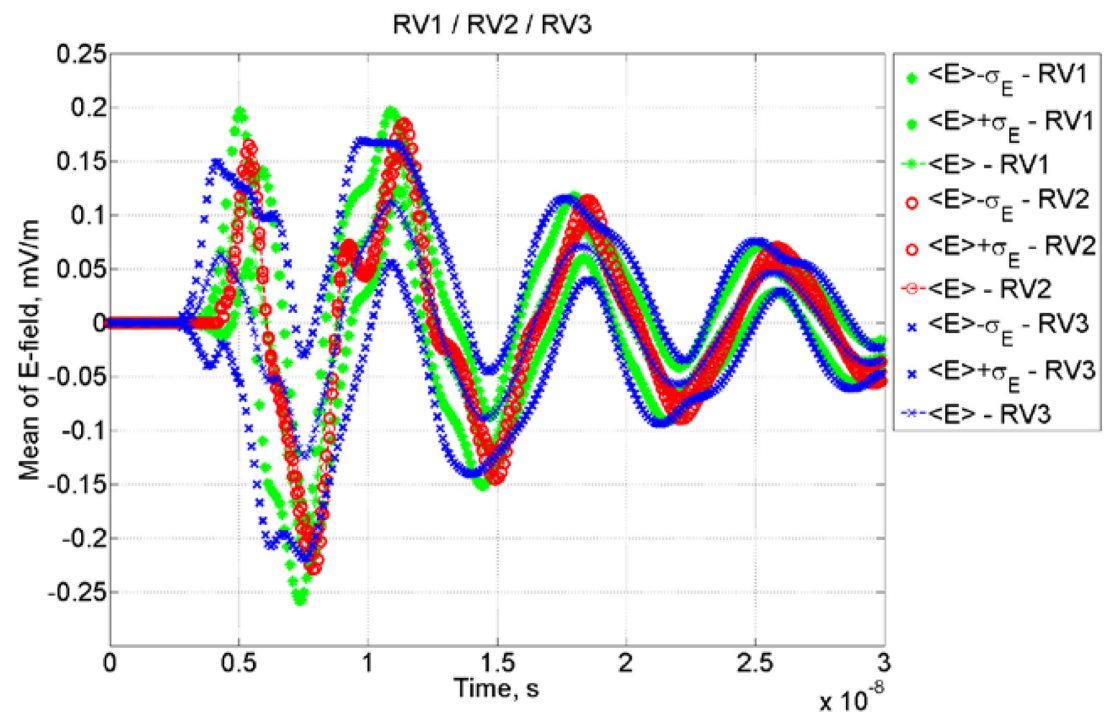

Figure 4: Mean of the $E$ - field inside soil, $L=1 \mathrm{~m}, a=6.74 \mathrm{~mm}$.

Finally, the influence of each RV to the output $E$ is considered using the following relation:

$$
\operatorname{Influence}(E)_{i}=\frac{\operatorname{Variance}\left(E \mid R V_{i}\right)}{\operatorname{Variance}(E \mid \text { tot })}
$$

Figure 5 shows the influence of each of three different RVs to the output of interest $E$.

Ranking random parameters from the most to the least influential (from $E$-field variances) it appears that one must primarily focus on RV3 (depth accuracy) and RV1 (antenna height), while the model is less sensitive to soil dielectric constant. Future work will include the influence of the conductivity which is a more demanding case within the deterministic modelling framework. 


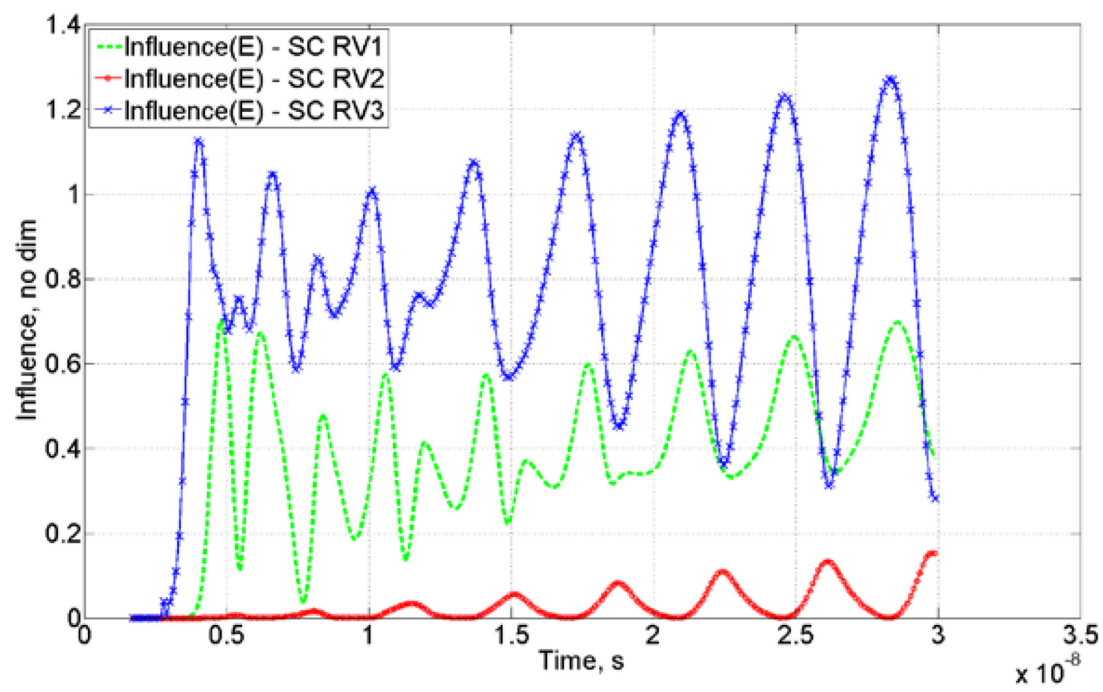

Figure 5: Influence of each RV to the output $E$.

\section{CONCLUDING REMARKS}

The paper presents deterministic stochastic analysis of the transient field generated by GPR dipole antenna and transmitted into the lower half-space by taking into account the uncertainty variations of the input data set. The deterministic model is based on the Hallen space-time integral equation formulation, integral formula for the transmitted field and the related boundary element solution procedures.

Having completed the deterministic modelling, simple SC formalism is applied to accurately account for uncertainties and to assess confidence intervals from the set of obtained numerical results.

The expansion of the statistical output in terms of mean and variance over a polynomial basis via SC has been shown to be a powerful method for uncertainty propagation and first-order sensitivity analysis demonstrating robustness, efficiency and providing satisfactory convergence rate and non-intrusiveness.

Future work on the improvement of system design is related to enhanced time domain formalism and increasing the number of random variables.

\section{ACKNOWLEDGEMENT}

This work benefited from networking activities was carried out within the EU funded COST Action TU1208 “Civil Engineering Applications of Ground Penetrating Radar."

\section{REFERENCES}

[1] Pajewski, L., Benedetto, A., Derobert, X., Giannopoulos, A., Loizos, A., Manacorda, G., et al., Applications of Ground Penetrating Radar in Civil Engineering - COST Action TU1208, 2013.

[2] Warren, C., Chiwaridzo, N. \& Giannopoulos, A., Radiation characteristics of a highfrequency antenna in different dielectric environments. 15th International Conference on Ground Penetrating Radar - GPR, Brussels, Belgium, pp. 796-801, 2014.

[3] Gürel, L. \& Oguz, U., Three-dimensional FDTD modeling of a ground-penetrating radar. IEEE Transaction on Geoscience and Remote Sensing, 38(4), 2000. 
[4] Cassidy, N.J. \& Millington, T.M., The application of finite-difference time-domain modelling for the assessment of gpr in magnetically lossy materials. Journal of Applied Geophysics, 67(4), pp. 296-308, 2009.

http://dx.doi.org/10.1016/j.jappgeo.2008.09.009

[5] Shangguan, P. \& Al-Qadi, I.L., Calibration of FDTD simulation of GPR signal for asphalt pavement compaction monitoring. Geoscience and Remote Sensing, IEEE Transactions on, 53(3), pp. 1538-1548, 2015.

[6] Poljak, D. \& Roje, V., Time domain calculation of the parameters of thin wire antennas and scatterers in a half-space configuration. IEE Proceeding Microwaves Antennas and Propagation, 145(1), pp. 57-63, 1998.

http://dx.doi.org/10.1049/ip-map:19981631

[7] Poljak, D., Tham, C.Y., McCowen, A. \& Roje, V., Transient analysis of two coupled horizontal wires over a real ground. IEE Proceding Microwave, Antnnas and Propagation, 147, pp. 87-94, 2000.

[8] Poljak, D., Advanced Modeling in Computational Electromagnetic Compatibility, John Wiley and Sons: New York, 2007.

http://dx.doi.org/10.1002/0470116889

[9] Poljak, D., Sesnic, S., Paric, D. \& El Khamlichi Drissi, K., Direct time domain modeling of the transient field transmitted in a dielectric half-space for GPR applications. In Electromagnetics in Advanced Applications (ICEAA), 2015 International Conference on, pp. 345-348, 2015.

http://dx.doi.org/10.1109/iceaa.2015.7297133

[10] Warren, C., Pajewski, L., Poljak, D., Ventura, A., Giannopoulos, A. \& Sesnic, S., A comparison of finite-difference, finite-integration, and integral-equation methods in the time-domain for modelling ground penetrating radar antennas, GPR 2016.

[11] Lallechere, S., Antonijevic, S., El Khamlichi Drissi, K. \& Poljak, D., Optimized numerical models of thin wire above an imperfect and lossy ground for GPR statistics. In Electromagnetics in Advanced Applications (ICEAA), 2015 International Conference on, pp. 907-910, 2015.

http://dx.doi.org/10.1109/iceaa.2015.7297246

[12] Dodig, H., Lallechere, S., Bonnet, P., Poljak, D. \& El Khamlichi Drissi, K., Stochastic sensitivity of the electromagnetic distributions inside a human eye modeled with a 3D hybrid BEM/FEM edge element method. Engineering Analysis with Boundary Elements, 49, pp. 48-62, 2014.

http://dx.doi.org/10.1016/j.enganabound.2014.04.005 